\title{
GAPS IN THE SATURATION SPECTRUM OF TREES
}

\author{
Ronald J. Gould \\ Department of Mathematics and Computer Science \\ Emory University, Atlanta USA \\ e-mail: rg@mathcs.emory.edu \\ PAUL HORN $^{1}$ \\ Department of Mathematics \\ University of Denver USA \\ e-mail: paul.horn@du.edu \\ Michael S. JACOBSON \\ Department of Mathematics \\ University of Colorado Denver, USA \\ e-mail: michael.jacobson@ucdenver.edu
}

AND

BREnT J. Thomas

Department of Mathematics and Statistics

Utah State University, USA

e-mail: brent.thomas@usu.edu

\begin{abstract}
A graph $G$ is $H$-saturated if $H$ is not a subgraph of $G$ but the addition of any edge from the complement of $G$ to $G$ results in a copy of $H$. The minimum number of edges (the size) of an $H$-saturated graph on $n$ vertices is denoted $\operatorname{sat}(n, H)$, while the maximum size is the well studied extremal number, $\operatorname{ex}(n, H)$. The saturation spectrum for a graph $H$ is the set of sizes of $H$-saturated graphs between $\operatorname{sat}(n, H)$ and $\operatorname{ex}(n, H)$. In this paper we show that paths, trees with a vertex adjacent to many leaves, and brooms have a gap in the saturation spectrum.
\end{abstract}

Keywords: saturation spectrum, tree, saturation number.

2010 Mathematics Subject Classification: 05C35, 05C05.

\footnotetext{
${ }^{1}$ This work was partially funded by NSA Young Investigator Grant H98230-15-1-0258.
} 


\section{REFERENCES}

[1] K. Amin, J. Faudree, R.J. Gould and E. Sidorowicz, On the non- $(p-1)$-partite $K_{p}$-free graphs, Discuss. Math. Graph Theory 33 (2013) 9-23. doi:10.7151/dmgt.1654

[2] C. Barefoot, K. Casey, D. Fisher, K. Fraughnaugh and F. Harary, Size in maximal triangle-free graphs and minimal graphs of diameter 2, Discrete Math. 138 (1995) 93-99.

doi:10.1016/0012-365X(94)00190- T

[3] G.A. Dirac, Some theorems on abstract graphs, Proc. Lond. Math. Soc. s3-2 (1952) 69-81. doi:10.1112/plms/s3-2.1.69

[4] P. Erdős, Extremal problems in graph theory, in: Proc. Sympos. Smolenice 13 (1963) $29-36$.

[5] P. Erdős, and M. Simonovits, A limit theorem in graph theory, Studia Sci. Math. Hungar. 1 (1965) 51-57.

[6] P. Erdős and T. Gallai, On maximal paths and circuits of graphs, Acta Math. Acad. Sci. Hungar. 10 (1959) 337-356.

[7] J. Faudree, R.J. Gould, M. Jacobson and B. Thomas, Saturation spectrum for brooms, manuscript.

[8] R.J. Gould, W. Tang, E. Wei and C. Zhang, The edge spectrum of the saturation number for small paths, Discrete Math. 312 (2012) 2682-2689. doi:10.1016/j.disc.2012.01.012

[9] W. Mantel, Problem 28, Wiskundige Opgaven 10 (1907) 60-61.

[10] A. McLennan, The Erdős-Sós conjecture for trees of diameter four, J. Graph Theory 49 (2005) 291-301.

doi:10.1002/jgt.20083

[11] A.F. Sidorenko, Asymptotic solution for a new class of forbidden r-graphs, Combinatorica 9 (1989) 207-215.

doi:10.1007/BF02124681

[12] P. Turán, On an extremal problem in graph theory, Mat. Fiz. Lapok 48 (1941) 436-452, in Hungarian.

[13] A. Doğan, On Saturated Graphs and Combinatorial Games (University of Memphis, 2016).

[14] J. Hladký, J. Komlós, D. Piguet, M. Simonovits, M. Stein and E. Szemerédi, The approximate Loebl-Komlós-Sós Conjecture I: The sparse decomposition, SIAM J. Discrete Math. 31 (2017) 945-982.

doi:10.1137/140982842 
[15] J. Hladký, J. Komlós, D. Piguet, M. Simonovits, M. Stein and E. Szemerédi, The approximate Loebl-Komlós-Sós Conjecture II: The rough structure of LKS graphs, SIAM J. Discrete Math. 31 (2017) 983-1016.

doi:10.1137/140982854

[16] J. Hladký, J. Komlós, D. Piguet, M. Simonovits, M. Stein and E. Szemerédi, The approximate Loebl-Komlós-Sós Conjecture III: The finer structure of LKS graphs, SIAM J. Discrete Math. 31 (2017) 1017-1071. doi: $10.1137 / 140982866$

[17] J. Hladký, J. Komlós, D. Piguet, M. Simonovits, M. Stein, M. and E. Szemerédi, The approximate Loebl-Komlós-Sós Conjecture IV: Embedding techniques and the proof of the main result, SIAM J. Discrete Math. 31 (2017) 1072-1148. doi:10.1137/140982878

Received 31 August 2016 Revised 26 June 2017 Accepted 26 June 2017 